\title{
Adaptive PI Control with Robust Variable Structure Anti-windup Strategy for Systems with Rate-limited Actuators: Application to Compression Systems
}

\author{
Yousif Eldigair ${ }^{\mathrm{a}, \mathrm{c}, *}$, Fabricio Garelli ${ }^{\mathrm{b}}$, Cristian Kunusch ${ }^{\mathrm{a}}$, \\ Carlos Ocampo-Martinez ${ }^{\mathrm{C}}$ \\ ${ }^{a}$ Brose Fahrzeugteile GmbH $\mathcal{E}$ Co., Würzburg, Germany \\ ${ }^{b}$ Institute LEICI, Universidad Nacional de La Plata - UNLP-CONICET, La Plata, \\ Argentina \\ ${ }^{c}$ Automatic Control Department, Universitat Politècnica de Catalunya, Institut de \\ Robòtica i Informàtica Industrial - CSIC-UPC, Barcelona, Spain
}

\begin{abstract}
In this paper, an adaptive proportional-integral (PI) control strategy for nonlinear systems with constrained actuators is discussed. In particular, a variable-structure anti-windup strategy that can be retrofitted onto different control structures is proposed and validated. Both the developed controller and anti-windup method are attuned to an automotive industrial control application: the control of air mass flow-rate and pressure in a fuel cell system using a high speed turbo-compressor and an electromechanical valve. Both the compressor and valve are rate-limited. The results from experimental tests agree with the simulations obtained and showing the effectiveness of the proposed approach by evidencing the fast control dynamics and the proper and desired closed-loop performance of the scheme without windup phenomena.
\end{abstract}

Keywords: sliding mode anti-windup, relay feedback test, air compressor, rate-limiter

\footnotetext{
${ }^{*}$ Corresponding author

Email address: yousif.eldigair@brose.com (Yousif Eldigair)
} 


\section{Introduction}

The limitation in actuators first derivative is recognized and widely accepted as a major control issue in high-end performance devices and industrial processes. This is the case of automotive electric drives, which nowadays require more accurate and aggressive performance in order to fulfill the demanding users requirements. When references to local controllers change, large and fast control actions are demanded to the actuators. The control actions are hindered by the rate-limitation of the actuators and the overall closed-loop control dynamics are therefore affected. Thus, the guarantee of system performance and closed-loop stability becomes critical [1]. In both fast and precise control tasks, rate-limitation becomes a stumbling block to achieve satisfactory system performance and stability, as it leads to the windup in either linear or nonlinear integral control schemes. Many strategies have been devised to counteract the controller windup caused by magnitude and rate-limitation $[2,3,4,5,6]$, particularly in mechanical, hydraulic and pneumatic actuators. However, rate-limitation constraints have drawn less attention in the case of electrical actuators, which are expected to be lighter, smaller, faster and more efficient.

One approach of handling both magnitude and rate saturations is through the use of model predictive controllers (MPCs). MPCs can intrinsically consider actuator limitations as constraints of the optimization problem embedded within the controller $[7,8,9,10,11,12,13]$. Accordingly, MPCs do not require any form of AW; rather, this is handled during the design phase of the controller all in one step through the addition of suitable constraints. As such, this approach of coping actuator constraints is also known as the one-step approach. MPCs have found applications in industrial fields and have drawn much attention in the academic community with numerous publications covering different types of preductive control laws $[14,15,16,17,18]$. While MPC can lead to a better overall performance than a conventional linear PI-controller, it introduces a significant amount of complexity and might increase the computational burden. The prime challenge in implementing MPC is the heavy numerical analysis that involves the solution of the associated optimization problem that is typically done online. While there are several studies demonstrating the effectiveness of MPC in nonlinear systems, the optimization algorithms associated are often far too complex for use in rudimentary micro-controllers such the electric control units (ECUs) related to automotive systems. In large nonlinear 
systems which require fast control dynamics, it would therefore be more feasible to devise two layers of control. At the lower layer, a conventional controller, for example a PI controller could be used. At the higher level, MPC can be used as a supervisory controller to generate reference signals for the lower layer controllers. In doing so, the computational requirement of the MPC can be reduced making it more feasible to implement in rudimentary ECUs.

Another approach of resolving the problem introduced by magnitude and rate-limitations is to augment a readily well-designed controller with an add-on AW scheme. This constitutes another step in addition to the design of the controllers. As such, this method of coping with actuator constraints is commonly referred to as the two-step approach. The simplicity of AW techniques relative to MPCs makes them more suitable for real-time implementation in automotive systems where the on-board computing power is quite limited. The main AW schemes for constrained systems can be generally classified into two classes [19].

The first class of AW strategies includes the conventional AW methods where the controller is modified, usually the integral term, such that the windup is suppressed. This is demonstrated in [20], where the design of an AW for both amplitude and rate saturation in the actuators is tackled. The system considered is represented with a linear model. The design problem was that of selecting the values of the AW gains and was formulated as a linear matrix inequality (LMI) optimization problem to maximize the region of asymptotic stability. This optimization is done only once offline, making this solution practical for implementation in automotive control units. This AW method was later on extended for sampled-data systems [21], reset control systems [22] and eventually linear parameter varying (LPV) systems [23] which can be used to model some of the nonlinearities in systems. It should be noted that in $[20,21,22]$, the AW was applied to conventional PI controllers. The results were satisfactory but the optimization problem can somewhat be challenging particularly when dealing with nonlinear systems.

In the second class, the reference signal to the control system is conditioned so as to respect the physical constraints of the process input. This latter procedure can be done conservatively by using either low-pass filters or rate-limiters at the reference. More effectively however, a signal can be injected and added to the reference such that the controller output does not violate the rate-limit constraint. Such AW schemes that apply reference conditioning are also know as reference governors [24, 25]. Numerous 
variations of reference governors are proposed in the literature. The most standard type of reference governors are scalar reference governors (SRG) [26]. Other more superior variants of reference governors are vector reference governors (VRGs) [27, 28] and command governors (CGs) [29, 30, 25], which can achieve faster control response than SRG. VRGs and CGs however, require that an optimization problem be solved in order to maximize the region of attraction or stability. Since the optimization in VRGs and CGs can be done offline, they are more practical for use in automotive applications than MPCs. Nevertheless, the optimization can still introduce significant complexity, particularly when nonlinear systems are taken into consideration. In [31, 19], a sliding mode controller (SMC) was proposed as means of reference governing. The robust properties of the SMC made the proposed SMC AW scheme fit for use in nonlinear systems. Furthermore, the AW method proposed did not require an optimization problem to be solved and therefore retained a simplistic structure.

It is noteworthy, that the presence of rate-limiters in closed-loop systems adds a considerable complexity to the tuning process of any PI controllers within the control loop. This is due to the fact that, unlike magnitude saturation, rate-limiters display dynamic properties that mask the dynamics of the actual plant. Relay-based tuning methods such as the Ästrom-Hägglund relay feedback test (RFT), explained in [32, 33, 34], would therefore be severely impacted by the phase shift and attenuation introduced by ratelimiters. As such, it is essential to analyze the dynamics of rate-limiters and determine their impacts on the oscillations when using relay-based tuning methods.

In this context, the core contribution and novelty of this paper stems from two attributes. First is the implementation of a sliding mode (SM)based AW strategy to a solve a real industrial problem. While the chattering phenomenon seen typically with SMCs generally makes them unfit for use in many industrial applications, this does not pose a problem with the proposed SM-based AW strategy since the discontinuity of the SMC signal is not passed on to the actuators. Due to the SMC utilized within AW strategy, it is more robust than conventional AW methods against system parameter variations and has no need for iterative re-tuning, making it ideal for nonlinear controllers and nonlinear systems as in the case of compression systems. Nonetheless, it retains a simplistic structure and is suitable for implementation in rudimentary automotive ECUs. The AW strategy can easily be added to lower layer controllers to handle rate-limitation constraints 
while having an MPC as a supervisory controller at the upper layer. The supervisory controller can be used to optimize of the overall system through yielding the optimal setpoints to the lower layer controller. It should be highlighted, however, that the prime focus of this paper is not on the upper layer control. Rather, the paper's objective is to improve on the performance of the lower level controller. The second point of contribution of this paper is the analysis of rate-limiters when applying a relay feedback. The analysis shows that the standard RFT tuning methodology cannot be used when dealing with rate-limited systems. The analysis was then used to develop a new modification to the standard relay-based identification for when rate-limiters exist in the system. The paper demonstrates the complete methodology from identification to PI-tuning and gain scheduling, all of which are based essentially on this modified RFT. This was done on actual system hardware with rate-limitations in two of its main actuators.

This paper is oriented towards an industrial application, which is to develop the controller for the mass flow rate and pressure of the air management system in fuel cell vehicles. The techniques covered can be used on different air management systems with different components. In addition, although methods and strategies presented were developed for automotive fuel cell applications, they can be implemented in other nonlinear systems with ratelimit and magnitude constraints. Preliminary results of the work done in this paper are presented in [35]. This paper builds on it by refining and simplifying the AW strategy and analyzing its reduced order dynamics. Furthermore, while [35] considers a single-input single-output system, this paper considers a multi-input multi-output compression system. Lastly, this paper supports the AW strategy and tuning methodology proposed via experimental results.

The remainder of this paper is organized as follows: Section 2 introduces the centrifugal air compression system and its control-oriented model (COM). Section 3 presents a novel anti-windup strategy for rate-limited systems based on first-order sliding mode (SM). The reduced-order SM dynamics were also analyzed in this section to show that, given a stable closed-loop system of PI controller and plant, the system retains stability when the proposed AW is included. Section 4 focuses on providing a comprehensive step by step PI tuning method for rate-limited systems based on analysis done using describing functions and the RFT approach. Each step is supported by simulation results. In Section 5, the simulation and testing results of the novel AW approach and associated PI tuning are presented. Finally, the conclusions of the research and development done are drawn and projections 


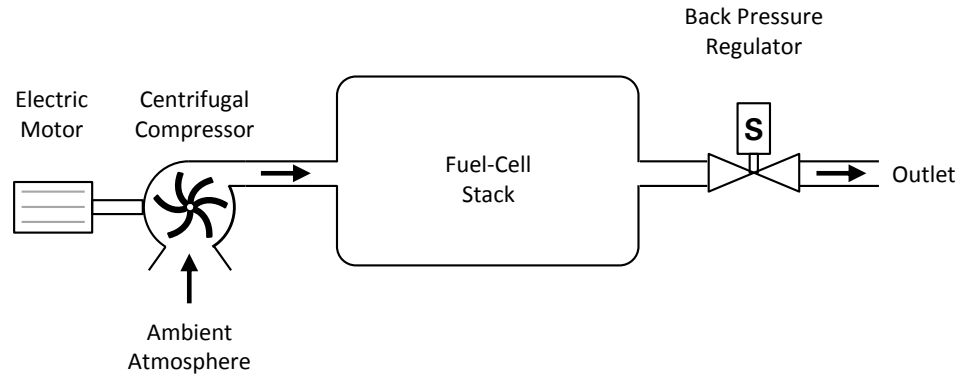

Figure 1: Compression system

of upcoming future works are mentioned in Section 6 .

\section{Compression System Description and Modeling}

In this section, the compression system depicted in Figure 1 is overviewed. It is important to remark that, since the tuning methodology, later discussed in this paper, relies on first identifying the system and then estimating the dynamics using a predetermined transfer function structure, the exact model is not of crucial importance. It is vital, however, to note the cross coupling between states and realize the nonlinearities within the system. The compression system is meant to emulate the behavior of the cathode module in a fuel cell vehicle.

\subsection{Electric Motor}

To drive the compressor, a three-phase permanent magnet synchronous motor (PMSM) is used. There are many control oriented models of PMSMs available in the literature as show in [36]. In many of these models, threephase currents are projected along two orthogonal axes ( $d q$-axes) through applying Park's transformation. In this fashion, the two currents $i_{d}$ and $i_{q}$ constitute two states of the PMSM. The third state is the mechanical speed $N_{c p}$ of the motor which associated with mechanical dynamics of first-order and is influenced by a disturbance. This disturbance is the mechanical load torque $T_{\text {load }}$ which is needed for the compression process. $N_{c p}$ introduces coupling nonlinearity into the electrical dynamics of the PMSM. Typically, Field-oriented control (FOC) is implemented to control $N_{c p}$. In FOC, three PI-controllers are introduced. The integral component of each of these controllers is associated with an additional state. As such, the motor can be represented as a six-state nonlinear system. 


\subsection{Compressor Maps}

The air mass flow rate, $W_{c p}(\mathrm{~kg} / \mathrm{s})$, at the outlet of the compressor is determined by the speed of rotation of the blades, $N_{c p}$, and the pressure ratio, $P R$, defined as the ratio of pressures from inlet $P_{c p}^{i n}$ to outlet $P_{c p}^{o u t}$

$\left(P R=P_{c p}^{o u t} / P_{c p}^{i n}\right)$. While it is possible to develop dynamic models of turbo compressors, such models require detailed knowledge of the boundary conditions at the inlet and outlet of the compressor [37,38]. These models also add complexity and demand heavy computational efforts; therefore, are unfit for control applications. Hence, the performances of centrifugal compressors are commonly studied through static turbo or compressor maps [39, 40, 41, 42]. Generally, the data of compression is provided in the form of look-up tables.

The compression process does not only raise the pressure but also the temperature of the air from the inlet $T_{a m b}$ to the outlet $T_{\text {out }}$ of the compressor such that $T_{\text {out }}>T_{\text {amb }}$. To evaluate the temperature $T_{\text {out }}$ at the outlet, the isentropic efficiency, denoted $\eta_{c p}$ must be known. $\eta_{c p}$ depends on $N_{c p}$ and $P R$ and is generally also provided in a look-up table.Furthermore, knowing the $\eta_{c p}$ and using thermodynamics principles, the demanded compression torque $T_{\text {load }}$ which acts on the motor can be evaluated.

\subsection{Gas Dynamics}

The gas dynamics of associated in the compression system are reflected on the pressure $P_{v}$ inside the volume of the fuel cell stack. The change in pressure is influenced by the inflow obtained from the compressor, which is $W_{c p}$, and the outflow $W_{\text {out }}$ through the control valve. Based on the characteristics of the valve, $W_{\text {out }}$ can be expressed as a nonlinear function of the inlet and outlet pressures and the temperature of air $T_{a}$ which can be assumed the same $T_{\text {out }}$ given the volume is small. $W_{\text {out }}$ is also determined by the valve travel, denoted $l_{v}$. Each $l_{v}$ leads to a respective valve discharge coefficient which can be used to control $W_{\text {out }}$, hence, the operation pressure within the stack. To control the pressure, a reference valve travel $l_{v}^{*}$ is given. While the relation between $l_{v}$ and $W_{\text {out }}$ is static, it is critical not to neglect the mechanical dynamics that govern the response of $l_{v}$ to step changes in the reference $l_{v}^{*}$. These dynamics can generally be estimated by a first-order transfer function. The pressure within the manifold then exhibits dynamics based on the difference between the in-flow rate $W_{c p}$ and out-flow rate $W_{\text {out }}$. 


\subsection{Overall System}

Combing all the aforementioned model descriptions, the overall two-input two-output compression system can be expressed in a compact form as

$$
\begin{aligned}
\dot{x}_{m} & =A\left(x_{m}\right) x_{m}+B u_{1}+\tilde{B} T_{\text {load }}\left(x_{g}, x_{m}\right), \\
\dot{x}_{g} & =\frac{R_{a}}{V} T_{a}\left(x_{m}, x_{g}\right)\left(W_{c p}\left(x_{m}, x_{g}\right)-W_{\text {out }}\left(x_{m}, x_{g}, x_{v}\right)\right), \\
\dot{x}_{v} & =\frac{1}{\tau_{v}}\left(x_{v}-u_{2}\right), \\
y_{1} & =W_{c p}\left(x_{m}, x_{g}\right), \\
y_{2} & =x_{g}
\end{aligned}
$$

where

$$
\begin{aligned}
& x_{m}=\left[\begin{array}{llllll}
i_{d} & i_{q} & N_{c p} & I_{1} & I_{2} & I_{3}
\end{array}\right]^{T}, \\
& x_{g}=P_{v} \text {, } \\
& x_{v}=l_{v} \text {, } \\
& u_{1}=N_{c p}^{*} \text {, } \\
& u_{2}=l_{v}^{*} \text {. }
\end{aligned}
$$

Here, $x_{m}, x_{g}$ and $x_{v}$ represent the states associated with the motor, the gas dynamics and the valve dynamics, $I_{1}, I_{2}$ and $I_{3}$ represent the states associated with integral terms of the three PI controllers used in FOC of the motor, $A\left(x_{m}\right)$ is a $6 \times 6$ matrix which depends on the motor parameters, such as the flux linkage, stator inductance and resistance, and can be expressed as a function of $N_{c p}$. $\tau_{v}$ denotes the time-constant of the valve, $R_{a}$ denotes the gas constant of air and $V$ denotes the overall lumped volume of the system from the compressor outlet to the control valve. Furthermore, $W_{c p}\left(x_{m}, x_{g}\right), W_{\text {out }}\left(x_{m}, x_{g}, x_{v}\right), T_{\text {load }}\left(x_{g}, x_{m}\right)$ and $T_{a}\left(x_{m}, x_{g}\right)$ are all nonlinear functions which depend on the states of the compression system.

The block diagram of the overall system is presented in Figure 2. The two inputs are essentially the references $N_{c p}^{*}$ and $l_{v}^{*}$ and the two outputs to be controlled are $W_{c p}$ and $P_{v}$. Three critical things are to be noted here. First, there are rate-limiters imposed on both inputs $u_{1}$ and $u_{2}$ due to actuator constrains in the motor and valve. Second, several nonlinearities exist within the system. Third, the dynamics of $W_{c p}$ and $P_{v}$ are not isolated; rather, are strongly coupled by the compressor maps and the pressure dynamics. 


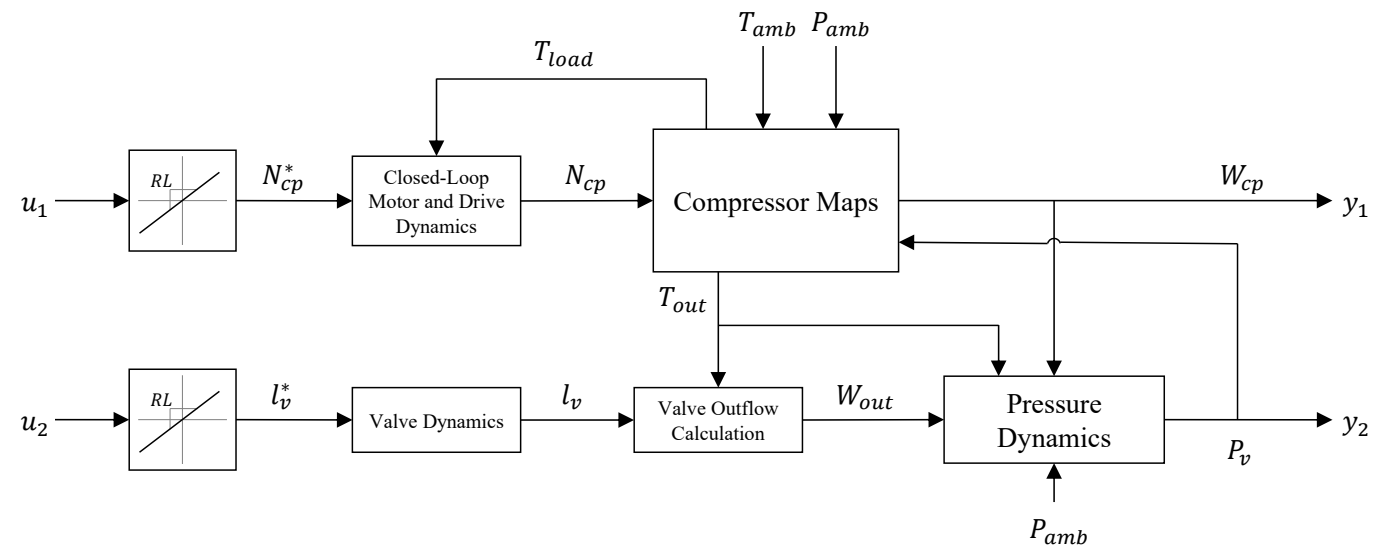

Figure 2: Block diagram of the overall compression system

\section{Variable Structure Anti-windup Strategy}

The considered compression system shown in Figure 1 is highly nonlinear due the compression map and valve outflow characteristics. Furthermore, the presence of rate-limited actuators implies that conventional AW methods would not be suitable $[6,43]$. Here, the variable structure AW strategy shown in Figure 3 is proposed. As can be seen, the proposal is based on a switching signal in order to take advantage of sliding mode robustness features.

The switching function $g(\sigma, e)$ is defined as

$$
w=g(\sigma, e)=\frac{1}{2}(1+\operatorname{sign}(\sigma e))=\left\{\begin{array}{cc}
1 & \text { if } \quad \sigma e \geq 0, \\
0 & \text { if } \quad \text { otherwise }
\end{array}\right.
$$

where $e$ is the output set-point tracking error and $\sigma$ denotes the sliding

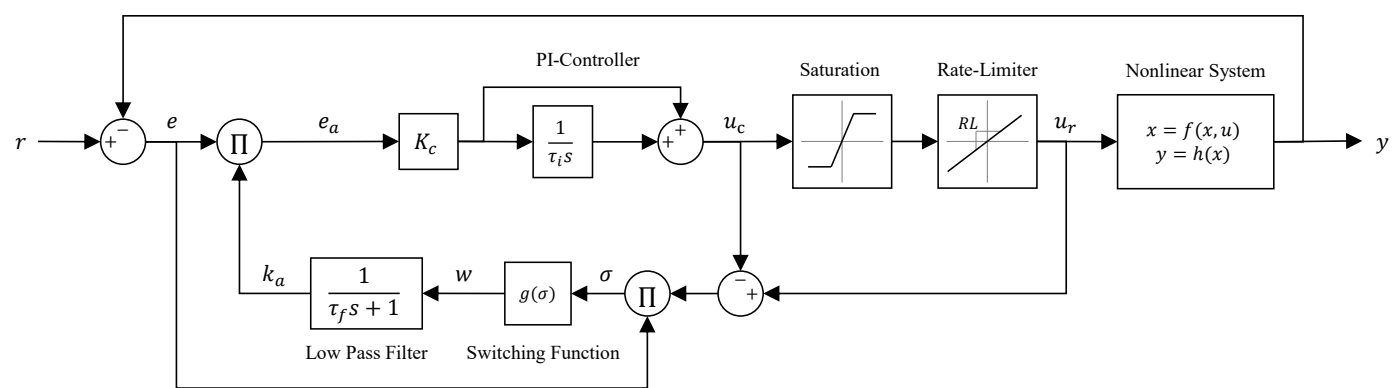

Figure 3: Proposed switching anti-windup method 
variable that is defined as

$$
\sigma=u_{r}-u_{c} .
$$

Here, $u_{c}$ is the controller output and $u_{r}$ is the control implemented after slew-rate and amplitude saturation.

The characteristics of the switching function drives the control loop into sliding mode when the rate-limiter is in effect. To prove that sliding mode is achieved, the dynamics introduced by the low-pass filter are first expressed as

$$
\dot{k}_{a}=\left(w-k_{a}\right) \frac{e}{\tau_{f}},
$$

where $w=g(\sigma, e)$ is the output of the switching function, $\tau_{f}$ is the time constant of the low-pass filter and $k_{a}$ is the output of the filter. The derivative of the sliding variable can then be computed as

$$
\begin{aligned}
\dot{\sigma} & =\dot{u}_{r}-\dot{u}_{c}, \\
& =\dot{u}_{r}-\left(\frac{e}{\tau_{i}}+\dot{e}_{a}\right) K_{c}, \\
& =\dot{u}_{r}-\left(\frac{e}{\tau_{i}} k_{a}+\dot{k}_{a} e+\dot{e} k_{a}\right) K_{c}, \\
& =\dot{u}_{r}-\left(\frac{e}{\tau_{i}} k_{a}+\left(w-k_{a}\right) \frac{e}{\tau_{f}}+\dot{e} k_{a}\right) K_{c}, \\
& =\dot{u}_{r}-\left(\frac{k_{a}}{\tau_{i}}+\left(w-k_{a}\right) \frac{1}{\tau_{f}}\right) K_{c} e-K_{c} k_{a} \dot{e},
\end{aligned}
$$

Here, $K_{c}$ and $\tau_{i}$ are the controller proportional gain and integral time constant. It should be emphasized that, assuming the amplitude saturation is not reached, the derivative $\dot{u}_{r}$ is restricted by the rate-limiter such that

$$
\frac{\mathrm{d} u_{r}}{\mathrm{~d} t}=\left\{\begin{array}{ccc}
\mathrm{RL}^{-} & \text {if } & u_{r}-u_{c}>0 \\
\mathrm{RL}^{+} & \text {if } & u_{r}-u_{c}<0 \\
\frac{\mathrm{d} u_{c}}{\mathrm{~d} t} & \text { if } & u_{r}-u_{c}=0
\end{array}\right.
$$

where $\mathrm{RL}^{-}$and $\mathrm{RL}^{+}$are constants that represent the negative and positive slew-rates of the rate-limiter respectively. Define now a positive constant $R L$ that satisfies $R L \leq\left|R L^{-}\right|$and $R L \leq\left|R L^{+}\right|$. Consider now the following cases:

- If $u_{r}-u_{c}>0$, then $\dot{u}_{r} \leq-\mathrm{RL}$ and $\sigma>0$ meaning $\sigma \dot{u}_{r} \leq-|\sigma| \mathrm{RL}$.

- If $u_{r}-u_{c}<0$, then $\dot{u}_{r} \geq+\mathrm{RL}$ and $\sigma<0$ meaning $\sigma \dot{u}_{r} \leq-|\sigma| \mathrm{RL}$.

- If $u_{r}-u_{c}=0$, then $\sigma \dot{u}_{r}=-|\sigma| \mathrm{RL}=0$. 
Essentially, the following inequality holds for all values of $\sigma$ :

$$
\sigma \dot{u}_{r} \leq-|\sigma| \mathrm{RL}
$$

The reachability condition is henceforth analyzed by evaluating

$$
\begin{aligned}
\dot{\sigma} \sigma & =\dot{u}_{r} \sigma-\left(\frac{1}{2 \tau_{f}}(\operatorname{sign}(e \sigma)+1)+\left(\frac{1}{\tau_{i}}-\frac{1}{\tau_{f}}\right) k_{a}\right) K_{c} e \sigma-K_{c} k_{a} \dot{e} \sigma \\
& \leq-\mathrm{RL}|\sigma|-\left(\frac{1}{2 \tau_{f}}(|e||\sigma|+e \sigma)+\left(\frac{1}{\tau_{i}}-\frac{1}{\tau_{f}}\right) k_{a} e \sigma\right) \frac{1}{\tau_{i}} K_{c}-K_{c} k_{a} \dot{e} \sigma .
\end{aligned}
$$

Consider now the case where $e \sigma \geq 0$. This essentially means that $e \sigma=|e||\sigma|$ holds and that

$$
\dot{\sigma} \sigma \leq-\mathrm{RL}|\sigma|-\left(1+\left(\frac{\tau_{f}}{\tau_{i}}-1\right) k_{a}\right) K_{c}|e||\sigma|-K_{c} k_{a} \dot{e} \sigma
$$

Assume also that $\dot{e}$ is bounded such that $|\dot{e}| \leq \Gamma$. In accordance with (6) (10), the sliding mode reachability condition for finite-time convergence can be expressed as

$$
\dot{\sigma} \sigma \leq \sigma f-|\sigma| \rho<-\eta|\sigma|,
$$

where $\eta$ is an arbitrary positive number that defines the reaching time and $f$ and $\rho$ are defined as

$$
\begin{aligned}
& f=-K_{c} k_{a} \dot{e}, \\
& \rho=\mathrm{RL}+\left(1+\left(\frac{\tau_{f}}{\tau_{i}}-1\right) \frac{k_{a}}{\tau_{f}}\right) K_{c}|e| .
\end{aligned}
$$

Considering that $0 \leq k_{a} \leq 1$ and given a small enough value of $\dot{e}$ in relation with RL, there exists a sufficiently small value of $\tau_{f}$ such that (11) is always satisfied for $e \sigma \geq 0$. This leads to the finite time convergence of $\sigma$ to 0 .

In the case where $e \sigma<0, e \sigma=-|e||\sigma|$ and (9) can be rewritten as

$$
\dot{\sigma} \sigma \leq-\mathrm{RL}|\sigma|+\left(\left(\frac{\tau_{f}}{\tau_{i}}-1\right) \frac{k_{a}}{\tau_{f}} K_{c}|e||\sigma|\right)-K_{c} k_{a} \dot{e} \sigma .
$$

Similarly, the reachability condition is expressed as

$$
\dot{\sigma} \sigma \leq \sigma f-|\sigma| \rho<-\eta|\sigma|
$$

where

$$
\begin{aligned}
& f=-K_{c} k_{a} \dot{e} \\
& \rho=\mathrm{RL}+\left(1-\frac{\tau_{f}}{\tau_{i}}\right) \frac{k_{a}}{\tau_{f}} K_{c}|e| .
\end{aligned}
$$


As with (11), (15) can be satisfied with a low $\tau_{f}$.

During the sliding phase where $\sigma=0$, the reduced-order dynamics of the system can be analyzed. The state-space representation of the low-pass filter and the PI-controller can be expressed as

$$
\begin{aligned}
& G_{f}(s)=\left\{\begin{array}{l}
\dot{x}_{f}=\lambda_{f} x_{f}+w, \\
k_{a}=x_{f},
\end{array}\right. \\
& G_{c}(s)=\left\{\begin{array}{l}
\dot{x}_{c}=A_{c} x_{c}+B_{c} e_{a}, \\
u_{c}=C_{c} x_{c}+D_{c} e_{a},
\end{array}\right.
\end{aligned}
$$

where $\lambda_{f}=-\frac{1}{\tau_{f}}$ represents the eigenvalue of the filter from (5) such that $\lambda_{f}<0, x_{c}$ represents the state introduced by the integral action of the PI controller, $A_{c}=0, B_{c}=\frac{K_{c}}{\tau_{i}}, C_{c}=1$ and $D_{c}=K_{c}$. The combined dynamics of the controller and anti-windup strategy becomes

$$
\begin{aligned}
{\left[\begin{array}{c}
\dot{x}_{c} \\
\dot{e}_{a}
\end{array}\right] } & =\left[\begin{array}{cc}
0 & B_{c} \\
0 & 0
\end{array}\right]\left[\begin{array}{l}
x_{c} \\
e_{a}
\end{array}\right]+\lambda_{f}(r-y)\left\{\left[\begin{array}{c}
0 \\
-\lambda_{f} x_{f}-x_{f} \frac{\dot{r}-\dot{y}}{r-y}
\end{array}\right]+\left[\begin{array}{c}
0 \\
w
\end{array}\right]\right\}, \\
u_{c} & =C_{c} x_{c}+D_{c} e_{a} .
\end{aligned}
$$

During sliding mode, $u_{c}=u_{r}$. Accordingly,

$$
e_{a}=D_{c}^{-1}\left(u_{r}-C_{c} x_{c}\right) \text {. }
$$

The reduced order dynamics of the controller can then be described by

$$
\dot{x}_{c}=Q_{c} x_{c}+B_{c} D_{c}^{-1} u_{r}
$$

where

$$
Q_{c}=A_{c}-B_{c} D_{c}^{-1} C_{c}
$$

The eigenvalues of the reduced-order dynamics are determined by the zeros of the controller transfer function as $\operatorname{eig}\left(Q_{c}\right)=\operatorname{zeros}\left(G_{c}(s)\right)$. Therefore, the hidden SM dynamics during the slew-rate compensation will be stable for any minimum-phase controller, particularly a PI controller. Hence, for a stable closed-loop system of PI controller and plant, the system remains stable once the AW strategy is fitted.

The developed AW strategy was tested in simulation for both positive and negative step changes in the mass flow reference. To demonstrate the flexibility of the AW strategy, the positive and negative slew rates were set 
to be different at $40 \mathrm{krpm} / \mathrm{s}$ and $-20 \mathrm{krpm} / \mathrm{s}$ respectively. As can be seen in Figure 4, the proposed AW method is successfully able to avoid the integral windup that would otherwise occur. This was seen for both the negative and positive step changes even though the slew rates are distinct. This indicates a virtuous feature of the proposed AW strategy, in that, no tuning needs to be done for different rate-limitations. Figure 4 also shows that the AW method is able to drive the sliding variable $\sigma$ to zero in finite time. The initial spike that can be seen at the start of the step change can be mitigated by filtering the reference flow rate with a relatively small time constant. If the time constant of this was chosen to be significantly lower than the time constant of the overall closed-loop system, its influence on the closed-loop dynamics can be minimized.

\section{Tuning of PI Controllers in Rate-limited Systems}

Optimal tuning of the PI controllers can be done to improve the performance of the closed-loop response in the instances where the rate-limiters are not active (not saturated). In this paper, optimal tuning using relay feedback test (RFT) approaches to obtain the controller parameters $K_{c}$ and $\tau_{i}$ is considered. There are three problems encountered when attempting to do so for the compression system shown in Figure 1.

Problem 1: The existence of a rate-limiter constraint associated with the actuators interferes with the outcome of the RFT. This is due to the fact that rate-limiters are dynamic constraints (affected by the frequency of the input signal) and essentially impose a phase lag within the control loop. This issue can be shown using the describing functions (DF) method

to analyze the standard Änstrom-Hägglund relay feedback system, discussed in $[32,33,34]$, when implemented in systems with rate-limiters as show in Figure 5.

For the forthcoming analysis, it will be assumed that the rate-limiter is always active at the limit cycle. This implies that the output signal $u_{r}$ of the rate-limiter is always triangular and never trapezoidal. While it is still possible to analyze the system in the case when $u_{r}$ is trapezoidal, it would require that an additional different describing function be derived. For the assumption that $u_{r}$ is triangular to hold, $u_{r}$ must always be in the band $\pm c$. This is generally particularly true for high frequency oscillations since $u_{r}$ becomes heavily attenuated by the rate-limiter and remains within the band $\pm c$. Lower frequency oscillations might require a higher $c$ due to the 

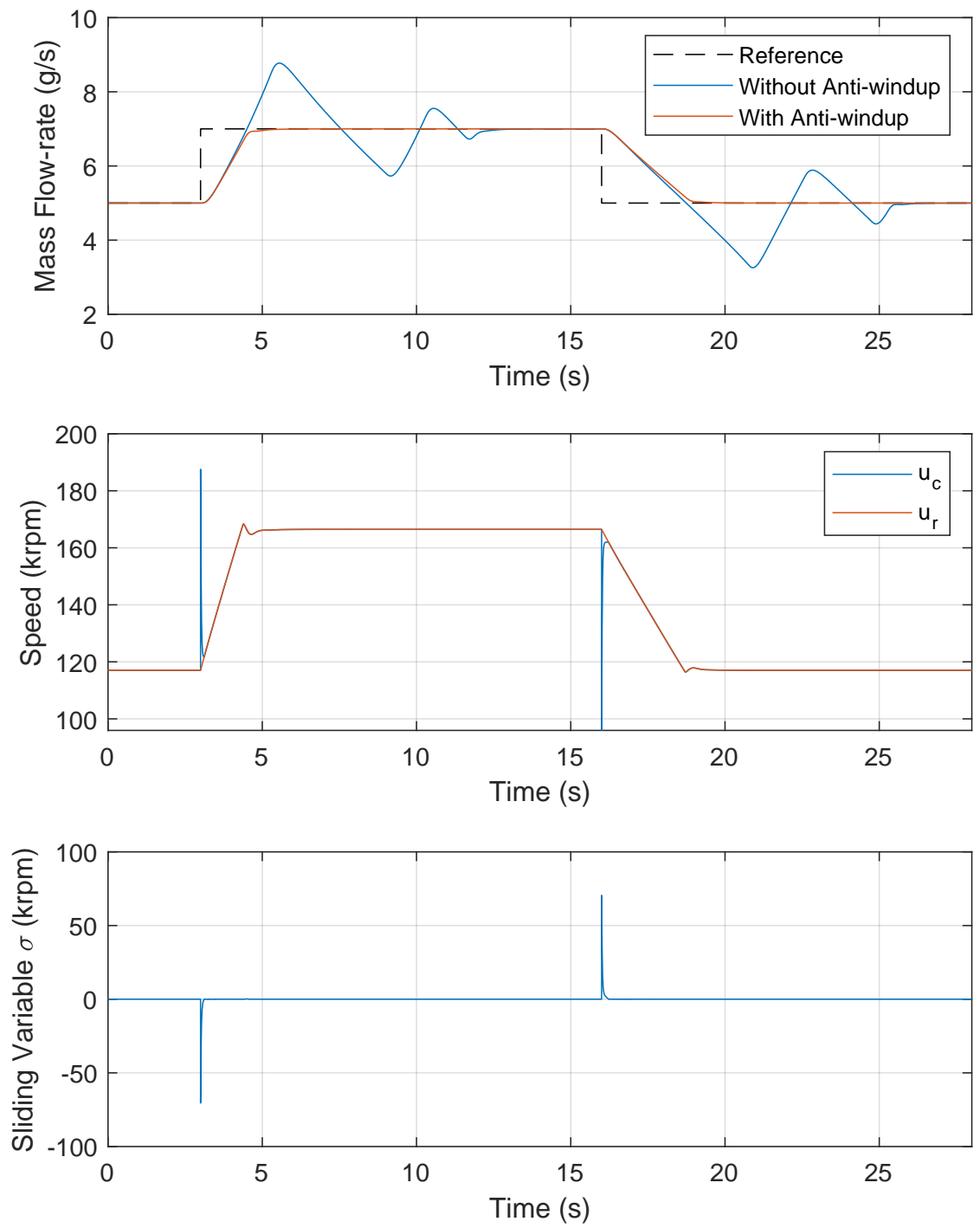

Figure 4: Simulated output response with and without the proposed AW method 


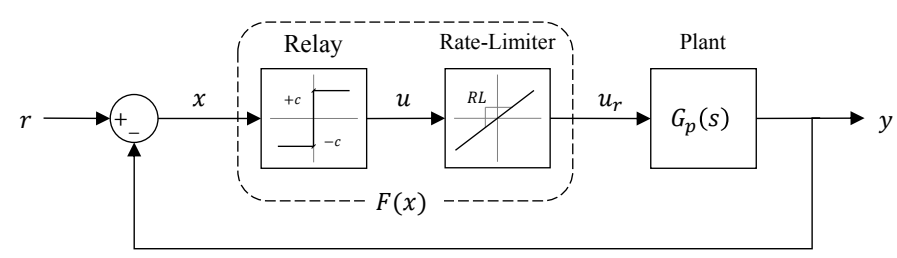

Figure 5: Standard RFT in a rate-limited system

lower attenuation caused by the rate-limiter. Another solution would be to reduce $\mathrm{RL}$ during the $\mathrm{RFT}$ which increase the attenuation and reduces the oscillations in $u_{r}$. This would avoid trapezoidal signals even if magnitude saturations exist within the loop and $c$ cannot be selected to be high enough.

The describing function $N(a, \omega)$ of the combined nonlinearity of the relay and rate-limiter can be derived as

$$
N(a, \omega)=p+j q
$$

where $a$ and $\omega$ are the amplitude and frequency of the oscillations in the output of the process and

$$
\begin{aligned}
& p=\frac{2}{\pi a} \int_{0}^{\pi} F(a \sin \psi) \sin \psi \mathrm{d} \psi \\
& q=\frac{2}{\pi a} \int_{0}^{\pi} F(a \sin \psi) \cos \psi \mathrm{d} \psi
\end{aligned}
$$

Here, $\psi$ denotes the phase of the sinusoidal input signal and $F(a \sin \psi)$ denotes the output of the nonlinearity of both relay and rate-limiter for a sinusoidal input signal $a \sin \psi$. The describing function can be expressed as

$$
\begin{aligned}
N(a, \omega) & =j \frac{4}{\pi a} \int_{0}^{\frac{\pi}{2}}\left(\frac{2 a_{r}}{\pi} \psi-a_{r}\right) \cos \psi \mathrm{d} \psi, \\
& =-j \frac{8}{\pi^{2}} \frac{a_{r}}{a}
\end{aligned}
$$

where $a_{r}$ is the amplitude of triangular oscillations in $u_{r}$ which is a function of both the slew-rate and frequency. For a large value of the relay output $\left(c>a_{r}\right)$, the amplitude is $a_{r}=\frac{\pi \mathrm{RL}}{2 \omega}$. Knowing $N(a, \omega)$, the harmonic balance equation (HBE), given by

$$
G_{p}\left(j \omega_{0}\right)=-N\left(a_{0}, \omega_{0}\right)^{-1}
$$




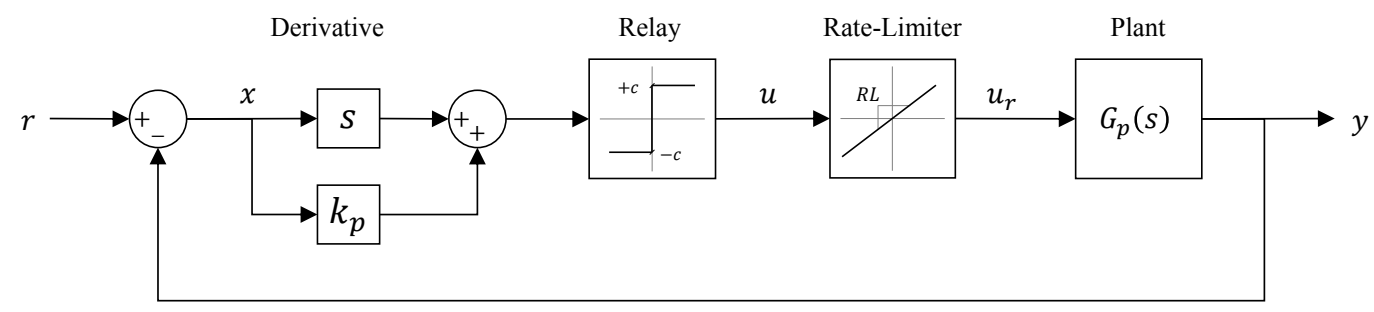

Figure 6: RFT with additional derivative term in rate-limited system

can be solved to evaluate $a_{0}$ and $\omega_{0}$, which denote the amplitude and frequency of the of self-sustained oscillations. As seen in $(28), N(a, \omega)$ is only imaginary only has no real component. As such, the oscillations excited by the relay are not at the crossover frequency where the phase is -180; rather, oscillation as excited with a phase of -90 . This provides little information about the gain margin and is therefor not enough to tune PI controllers to achieve a certain gain or phase margin. Hence, the standard RFT is not suitable for use in systems with rate-limiters.

To account for the phase shift introduced by the rate-limiter, the RFT test shown in Figure 6 can be used. By introducing an additional derivative term, limit cycles can be excited at an angle $\phi$ from the negative real axis. This is due to the phase shift introduced by the derivative term, which can be calculated as

$$
\phi=\arctan \left(\frac{k_{p}}{\omega}\right),
$$

where $k_{p}$ is the gain of the proportional channel as depicted in Figure 6 . The describing function of the overall nonlinearity of both the relay and rate-limiter can derived using (25) - (27) as

$$
\begin{aligned}
N(a, \omega) & =\frac{4 \mathrm{RL}}{\pi a \gamma}\left(1-j \frac{k_{p}}{\omega}\right), \\
-N^{-1}(a, \omega) & =-\frac{\pi a \gamma}{4 \mathrm{RL}}\left(1+j \frac{\omega}{k_{p}}\right),
\end{aligned}
$$

where

$$
\gamma=\sqrt{\omega^{2}+k_{p}^{2}}
$$

The magnitude of $N(a)$ is also evaluated as

$$
|N(a, \omega)|=\left|\frac{4 \mathrm{RL}}{\pi a \omega}\right| .
$$




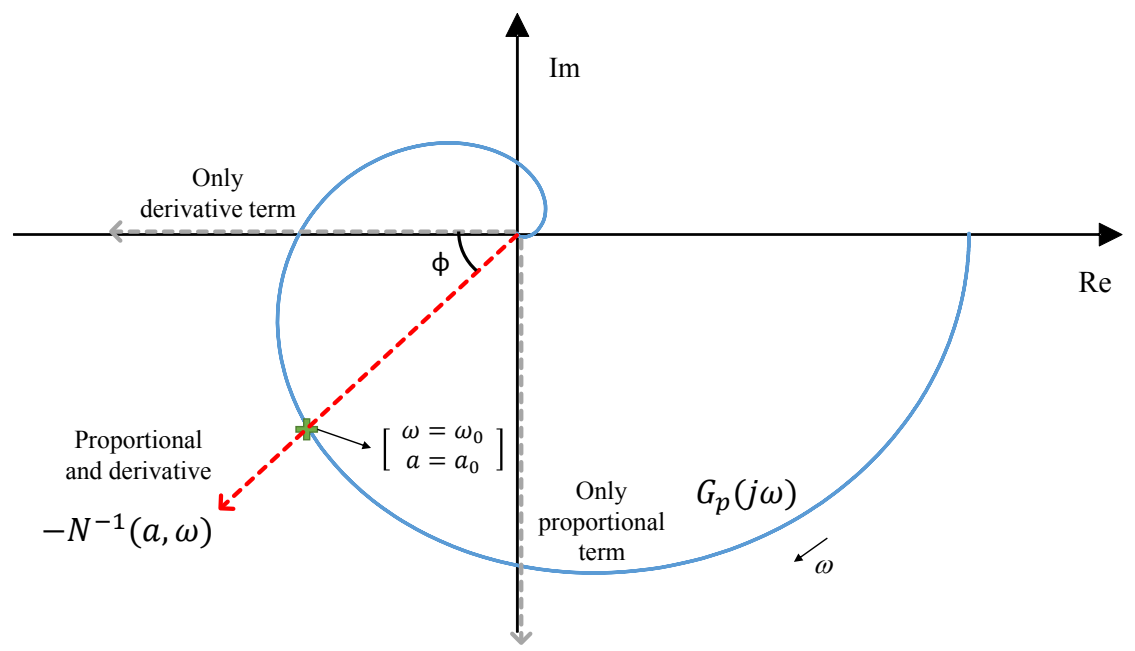

Figure 7: Graphical interpretation of RFT with derivative term in rate-limited system

Algorithm 1: The RFT with the derivative term shown in Figure 6 is implemented. By selecting different values of $k_{p}$, oscillations are excited with different $\omega_{0}$ and $a_{0}$ in the output. Both $\omega_{0}$ and $a_{0}$ are measured. $\omega_{0}$ is then used to calculate $\phi$ as shown in (30) and $a_{0}$ is used to find the magnitude of the phasor $\left|G_{p}\left(j \omega_{0}\right)\right|=\left|N\left(a_{0}, \omega_{0}\right)\right|^{-1}$ using (33). Through doing so, the system can be identified. A graphical interpretation of this algorithm is show in Figure 7.

Problem 2: The system is of multi-input multi-output nature. The two control inputs are the compressor speed reference $N_{c p}^{*}$ and the valve position reference $l_{v}^{*}$. The two system outputs are the mass flow $W_{c p}$ and pressure $P_{v}$. Since the states are coupled, the PI controller used for $W_{c p}$ influences the dynamics of $P_{v}$ and vice-versa.

Algorithm 2: In this paper, $N_{c p}^{*}$ is to be used to control $W_{c p}$. This control loop will be referred to as loop 1 . In loop $2, l_{v}^{*}$ is be used to control $P_{v}$. To autotune this MIMO system, a sequential approach is utilized following these steps:

1. Using non-optimally tuned PI controllers, the system is taken to a certain operating point of pressure and mass flow.

2. The valve position, $l_{v}^{*}$, is then held constant while the RFT proposed in Figure 6 is implemented in loop 1.

3. Given certain homogeneous tuning laws $c_{1}$ and $c_{2}$, the gain $k_{p}$ is changed 
until oscillations are created at an angle

$$
\phi_{c}=\arctan \left(\frac{1}{2 \pi c_{2}}\right)
$$

The oscillations are created at an angle $\phi_{c}$ rather than the negative real axis to account for the phase shift introduced by the integral action of the PI controller. The $\omega_{0}$ and $a_{0}$ of oscillations in $W_{c p}$ are measured and the PI control parameters for loop 1 are calculated following

$$
K_{c}=c_{1} \frac{4 \mathrm{RL}}{\pi a_{0} \omega_{0}} \quad, \quad \tau_{i}=c_{2} \frac{2 \pi}{\omega_{0}}, \quad K_{i}=\frac{K_{c}}{\tau_{i}}
$$

where RL is the rate-limit used for the speed during the RFT and the tuning laws $c_{1}$ and $c_{2}$ are associated with loop 1 .

4. A RFT is implemented next with the relay in loop 2 and using the PI parameters for loop 1 obtained in the previous step. The test is used to obtain PI parameters for loop 2 using (35) but with a different set of tuning rules $c_{1}$ and $c_{2}$ and using the value of RL used in the RFT of loop 2 .

5. A RFT is then implemented in loop 1 while using the PI parameters of loop 2 obtained in the previous step.

6. Steps 3 and 4 are repeated until the PI parameters for both loops converge.

Problem 3: The system is nonlinear. This essentially means that it must be identified at several different operating conditions to generate a suitable set of tuning laws $\left(c_{1}\right.$ and $\left.c_{2}\right)$ for both loops 1 and 2 . The RFT must then be done at different operating conditions and a schedule can then be used for the controller gain $K_{c}$ and the integral time constant $\tau_{i}$ while incorporating the AW strategy presented in Figure 3.

To develop process-specific homogeneous tuning laws, system identification is done using the RFT at different pressures and mass flow-rates. Since there are two inputs and two outputs, there are four transfer functions which can be obtained. The Nyquist plots of these transfer functions are shown in Figure 8 . The plots for both loop 1 and loop 2 can be approximated by a second-order plus dead time (SOPDT) model, given by

$$
G_{p l}(s)=\frac{K_{p l} e^{-s \theta}}{\tau_{p l}^{2} s^{2}+2 \xi \tau_{p l} s+1},
$$



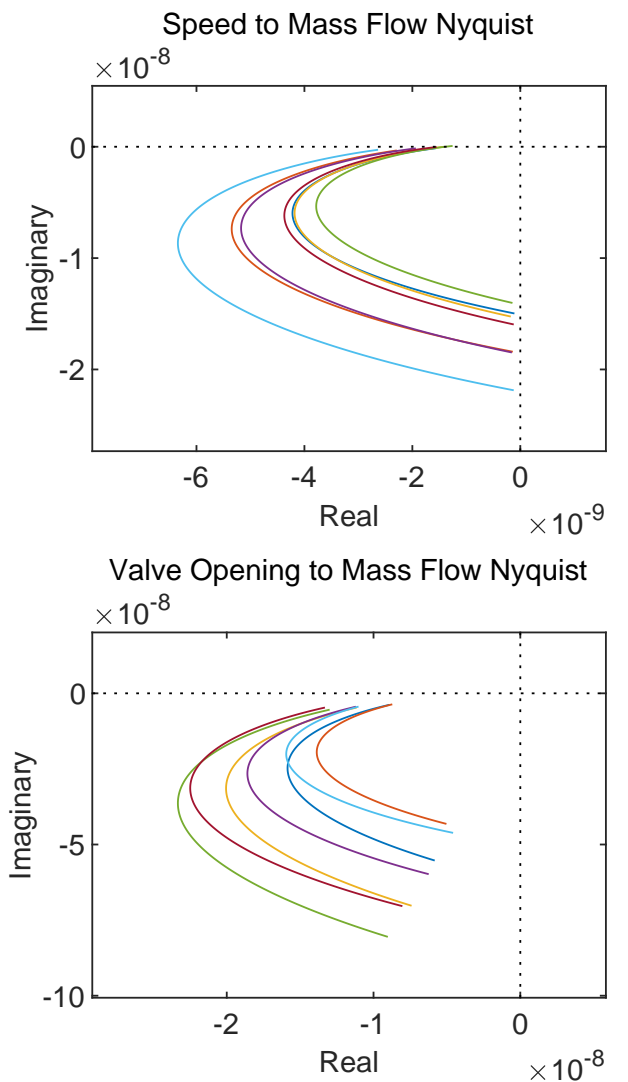

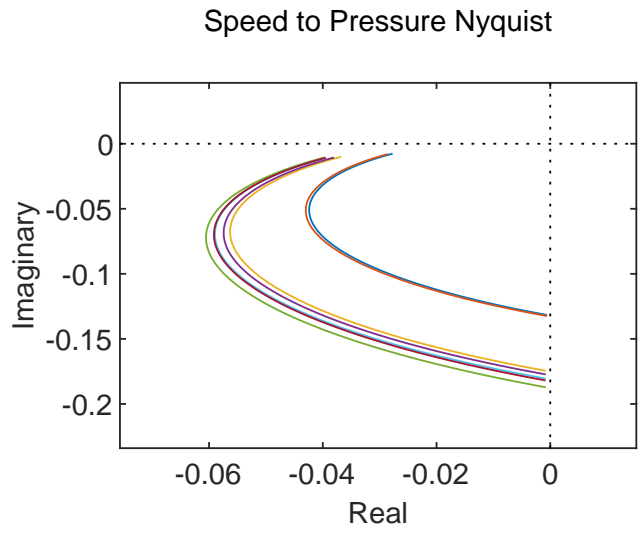

Valve Opening to Pressure Nyquist

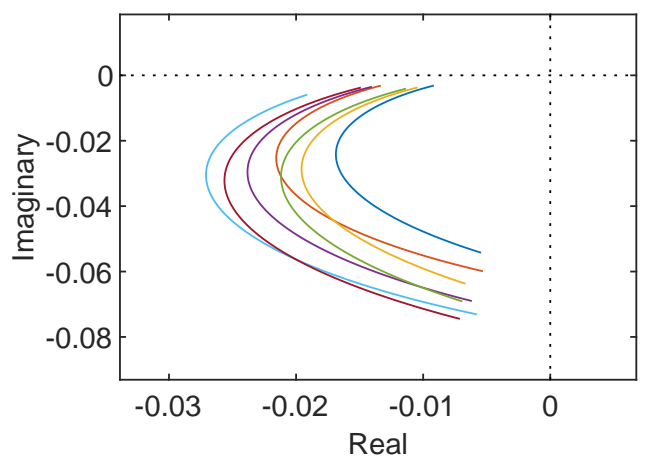

Figure 8: Nyquist plots of system in 7 different operating conditions
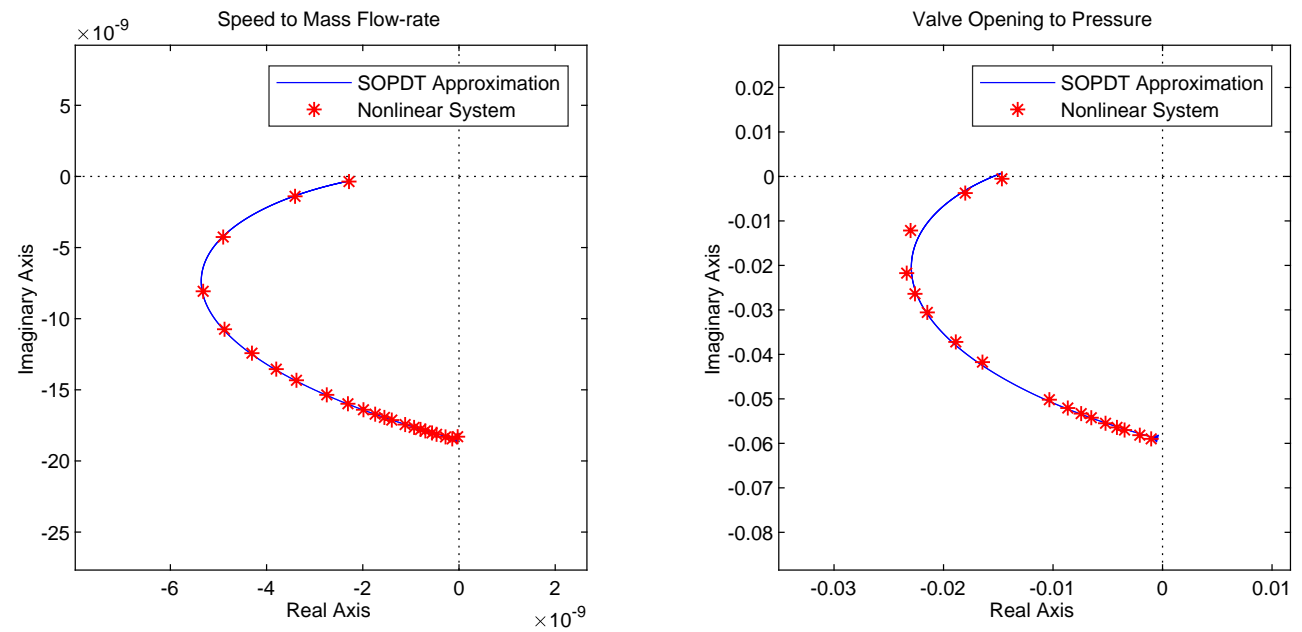

Figure 9: Identification of loops 1 and 2 at $W_{c p}=6 \mathrm{~g} / \mathrm{s}$ and $P R=1.2$ 
where the plant gain $K_{p l}$ and time constant $\tau_{p l}$ vary based on the operating condition.

The operating point where $W_{c p}=6 \mathrm{~g} / \mathrm{s}$ and the pressure ratio $P R=1.2$ will be considered. At this operating condition, the Matlab Curve Fitting Toolbox can be used to identify the plant parameters. Table 1 displays the obtained parameters of loops 1 and 2. As can be seen in Figure 9, the parameters provide a suitable estimate.

Table 1: Results of system identification at $W_{c p}=6 \mathrm{~g} / \mathrm{s}$ and $P R=1$
\begin{tabular}{|c|c|c|}
\hline & Loop 1 & Loop 2 \\
\hline Transfer function paramters: & $3.649 \times 10^{-8}$ & 0.1001 \\
$K_{p l}$ & 0.1193 & 0.0834 \\
$\tau_{p l}$ & 1.0906 & 1.1226 \\
$\xi$ & 0.0331 & 0.0130 \\
$\theta$ & & \\
\hline Optimal tuning laws (IAE): & 0.3283 & 0.3321 \\
$c_{1}$ & 0.9044 & 1.8193 \\
$c_{2}$ & 10.0 & 5.0 \\
$\theta_{c}$ & \multicolumn{2}{|}{}
\end{tabular}

For a class of SOPDT systems with known values of $\frac{\theta}{\tau_{p l}}$ and $\xi$, tuning laws can be proposed to optimize the closed-loop response, such that the integral absolute error (IAE) or the integral time-weighted square error (ITSE) is minimum [33]. Minimization of the IAE or the ITSE leads to fast convergence of the error while penalizing and reducing overshoots. This is fact crucial in fuel cell systems to avoid high pressure peaks and reduce unnecessary fast fluctuations in the compressor speed which could be damaging to the compressor and lead to system inefficiencies. Furthermore, since optimal tuning rules also incur fast set-point tracking, proper overall control performance can be achieved. A constraint to attain a predefined closed-loop gain margin or phase margin can be set to this optimization problem. In this paper, the IAE is considered and a constraint is set to have a closed-loop gain margin of 3. The tuning laws to achieve this for loops 1 and 2 are give in Table 1 . These tuning laws hold for all operating conditions since the ratio $\frac{\theta}{\tau_{p l}}$ and the parameter $\xi$ remain almost the same throughout all operating conditions. The variations in the Nyquist plots shown in 8 are mostly due to variations in the parameters $K_{p l}$ and $\tau_{p l}$. 
Algorithm 3: The processes for loops 1 and 2 are identified at one operating condition. Homogeneous tuning laws are obtained from the identified processes. The sequential tuning algorithm discussed earlier is now followed to obtain the PI parameters for both loops. This is done at different operating conditions to eventually construct a gain schedule for the PI parameters.

Following the aforementioned algorithms, tuning of the compression system was done at different operating conditions. Tables 2 to 5 in the appendix display the values obtained from the simulation done. Polynomial functions were used to fit the given data as to obtain smooth continuously differentiable functions of the control parameters. These functions for loop 1 are

$$
\begin{aligned}
K_{c}\left(W_{c p}, P R\right) & =\left(-3.79-443 W_{c p}+6.56 P R\right) \times 10^{8}, \\
K_{i}\left(W_{c p}, P R\right) & =\left(-4.94-396 W_{c p}+6.75 P R\right) \times 10^{9},
\end{aligned}
$$

and for loop 2 are

$$
\begin{aligned}
K_{c}\left(W_{c p}, P R\right) & =\left(2.48+38.3 W_{c p}+1.80 P R\right) \times 10^{-4} \\
K_{i}\left(W_{c p}, P R\right) & =\left(1.92-28.1 W_{c p}-1.38 P R\right) \times 10^{-4}
\end{aligned}
$$

Figure 10 shows simulation results that illustrate the key features of the presented control topology. It compares results between a PI-controller without AW that is manually tuned to reduce the windup effect and an optimally tuned PI controller (optimized at $W_{c p}=6 \mathrm{~g} / \mathrm{s}$ and $\mathrm{PR}=1.2$ ) with the proposed AW method. Figure 10 also illustrates the performance of the adaptive PI strategy implemented using (37) - (40). It can be seen that when no AW strategy is incorporated, the system suffers high integral windup as demonstrated by the high overshoot in the mass flow-rate at the initial positive reference step change, leaving the algorithm unsuitable for use in industrial applications. The controller gains can be further reduced to decrease the overshoot due to integral windup but this would eventually lead to a slower closed-loop performance. When the proposed AW strategy is implemented, the integral windup is eliminated. However, without gainscheduling, the closed-loop response is unstable at some operating conditions as seen at high mass flow-rates and pressures. The proposed adaptive PI with AW is able to retain successful tracking of the set-points of the mass flow and pressure ratio throughout the different operating conditions. The prime benefit of the proposed AW method is that it can be coupled to any nonlinear controller with integral action and without the need for re-tuning. 

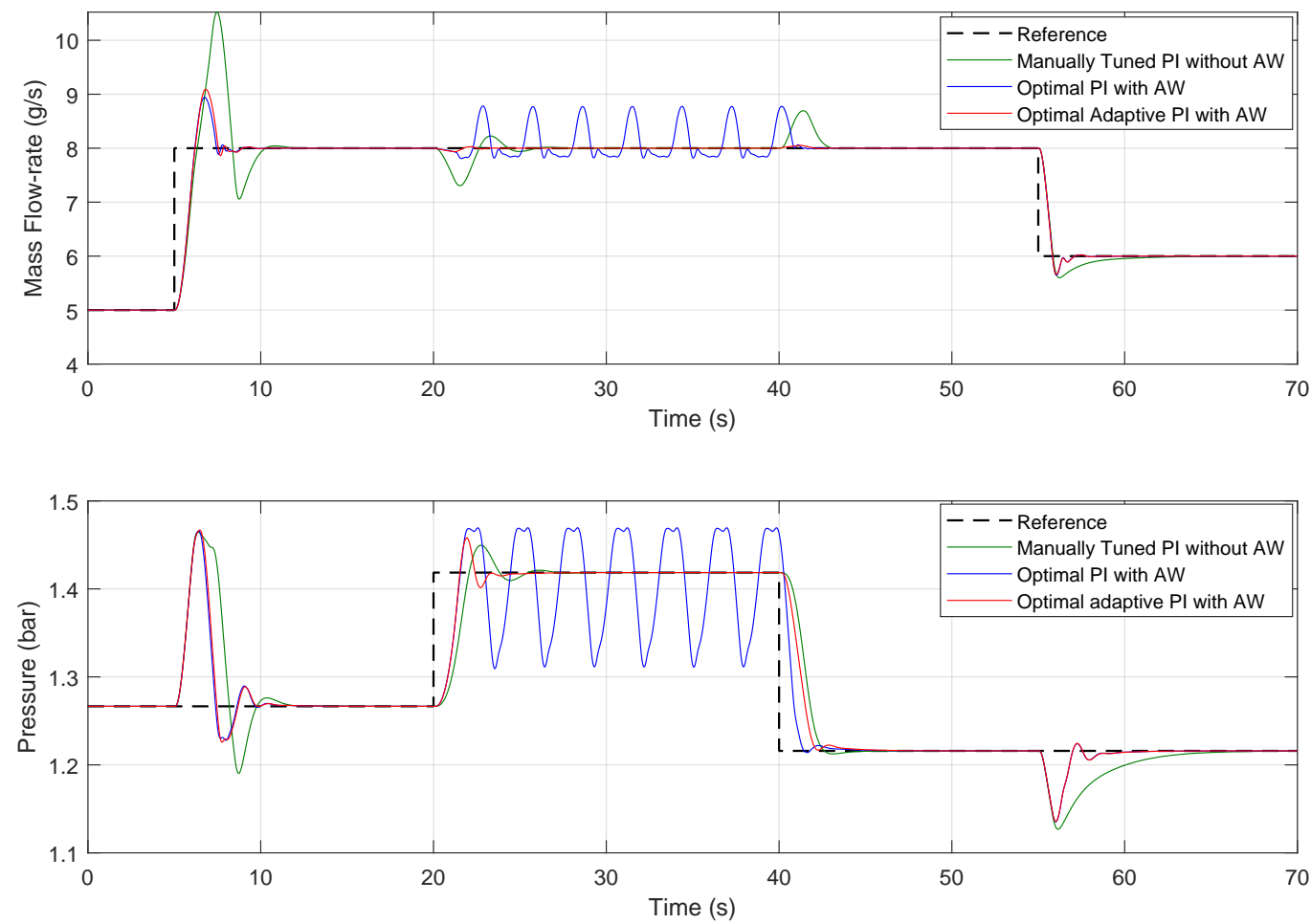

Figure 10: Comparison of the performance of different control schemes

This makes the proposed method suitable for its direct use in industrial applications with already existing control/regulation modules. The use of adaptive PI schemes with the AW strategy shows promising results, providing a systematic methodology able to be directly implemented in different nonlinear industrial processes with rate-limitation.

\section{Experimental Results}

As to validate the proposed AW strategy in Section 3 and tuning methodology proposed in Section 4 experimentally, the hardware setup shown in Figure 11 was developed.

\subsection{Variable Structure Anti-windup}

The proposed AW in Figure 3 was tested on a hardware setup. A PI controller was used in loop 1 and the closed-loop responses for a positive and a negative step change in the reference with and without the AW were 


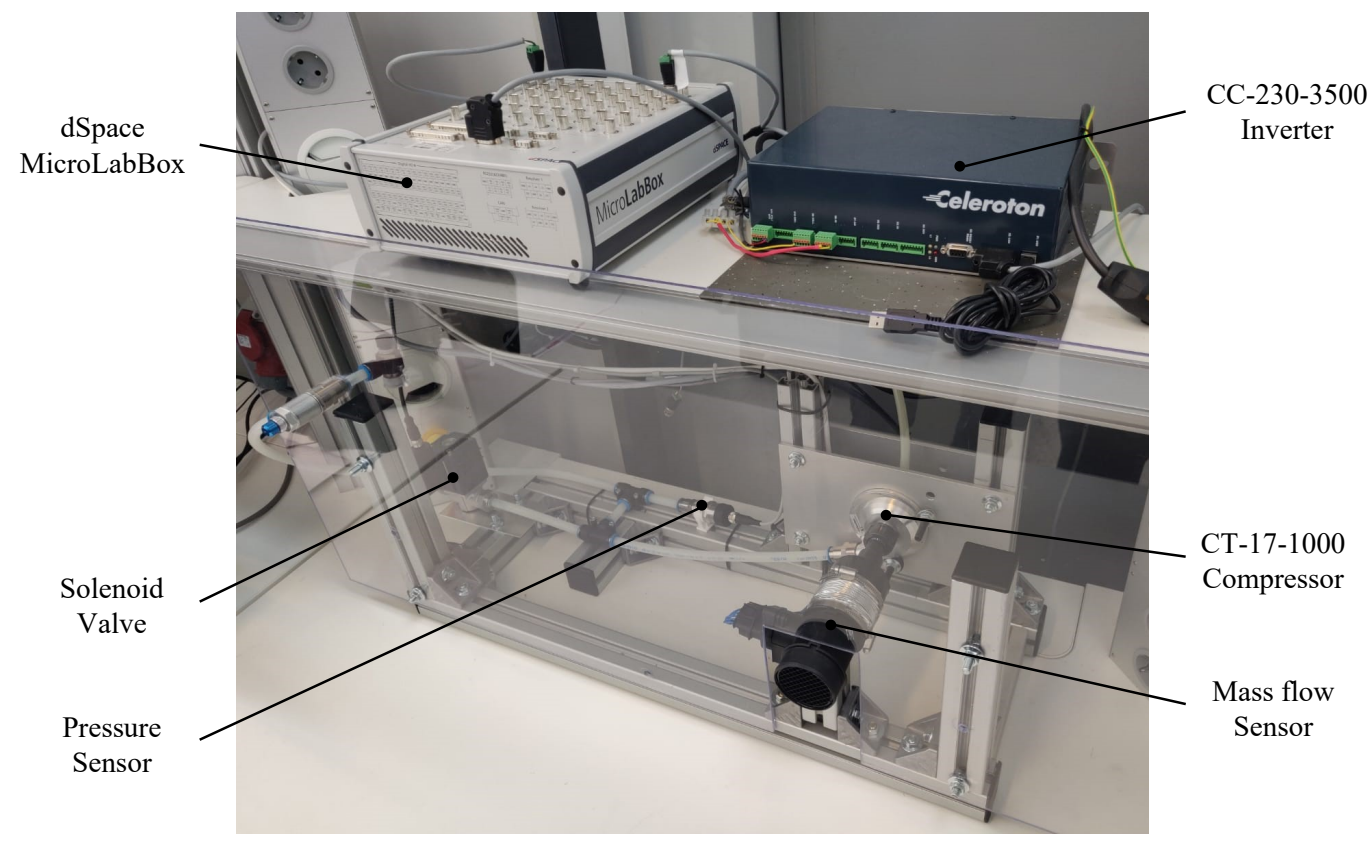

Figure 11: Hardware setup

measured. The acceleration and deceleration were set to be different at $40 \mathrm{krpm} / \mathrm{s}$ and $-20 \mathrm{krpm} / \mathrm{s}$. Figure 12 presents the experimental results obtained.

As illustrated in Figure 12, without AW, the closed-loop response suffers high over/undershoots, which agrees with the simulation results in Section 3. With the proposed AW, control of the mass flow-rate was achieved relatively fast without any over/undershoots despite the difference between the positive and negative slew-rates and the nonlinearity of the system.

\subsection{Adaptive PI Controller}

Following Algorithm 1, the RFT scheme proposed in Figure 6 was implemented in both loops 1 and 2 at several operating conditions. Figure 13 shows the results obtained with $W_{c p}=6 \mathrm{~g} / \mathrm{s}$ and $P_{v}=1.2 \mathrm{bar}$. As illustrated, SOPDT leads to proper approximation of the response obtained experimentally.

The optimal tuning parameters for the minimum IAE and a gain margin of 3 are $c_{1}=0.3205$ and $c_{2}=0.5563$ for loop 1 and $c_{1}=0.3301$ and $c_{2}=$ 1.1321 for loop 2. The phase shifts introduced by the tuning laws for loops 


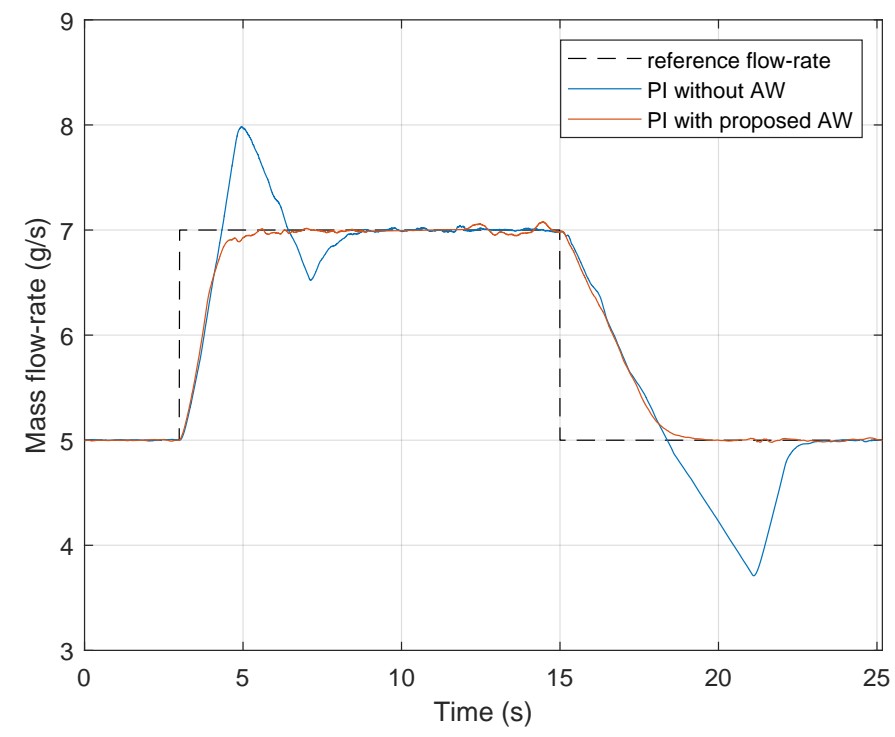

Figure 12: Experimental results of the AW method in the mass flow-rate control loop

1 and 2 are $\phi_{c}=15.97^{\circ}$ and $\phi_{c}=8.00^{\circ}$, respectively. Knowing the tuning laws for both loops, Algorithm 3 was followed and used to construct a gain schedule for the PI controller parameters. The parameters are given in Tables 6 to 9 in the appendix. The following functions were used to fit the data displayed in those tables and create an adaptive PI control structure for loop 1:

$$
\begin{aligned}
K_{c}\left(W_{c p}, P_{v}\right)= & 1.10 \times 10^{8}-2.61 \times 10^{10} W_{c p} \\
& +231 P_{v}-2.10 \times 10^{4} W_{c p} P_{v}+1.73 \times 10^{12} W_{c p}{ }^{2}, \\
K_{i}\left(W_{c p}, P_{v}\right)= & -7.92 \times 10^{9}-7.49 \times 10^{11} W_{c p} \\
+ & 1.67 \times 10^{5} P_{v}+5.24 \times 10^{6} W_{c p} P_{v}-0.775 \times 10^{12} P_{v}{ }^{2},
\end{aligned}
$$

and for loop 2

$$
\begin{aligned}
& K_{c}\left(W_{c p}, P_{v}\right)=2.64 \times 10^{-4}+2.06 \times 10^{-3} W_{c p} \\
&- 2.93 \times 10^{-9} P_{v}+3.05 \times 10^{-7} W_{c p} P_{v}-2.93 W_{c p}{ }^{2}, \\
& K_{i}\left(W_{c p}, P_{v}\right)= 3.22 \times 10^{-4}+7.63 \times 10^{-2} W_{c p} \\
&-6.29 \times 10^{-9} P_{v}+5.80 \times 10^{-7} W_{c p} P_{v}-10.35 W_{c p}{ }^{2} .
\end{aligned}
$$



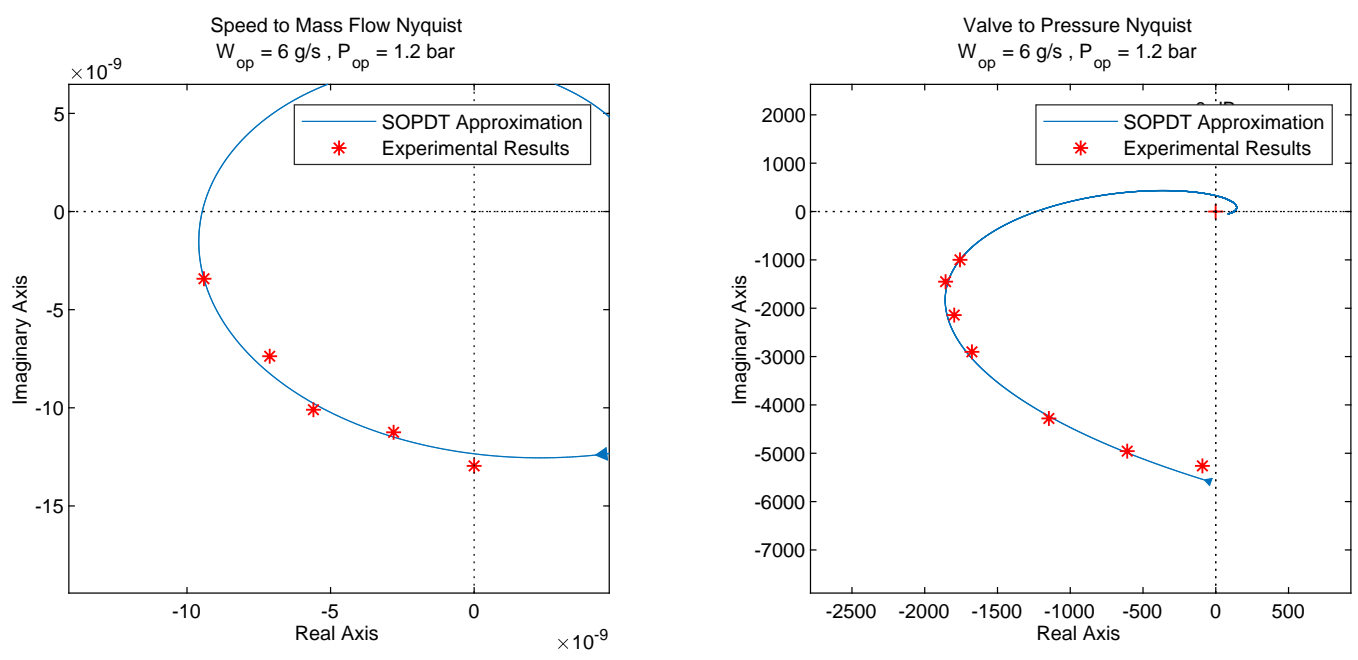

Figure 13: Experimental system identification using proposed RFT

The overall MIMO system with the adaptive PI controllers and the proposed AW method were tested for step changes in the reference flow-rate and pressure. The results are displayed in Figure 14. It can be seen that the experimental results agree with the simulation results in Figure 10 in the sense that integral windup for both the mass flow and pressure controllers is avoided while retaining fast control performance. This experimental result effectively proves the viability of the AW strategy and tuning method proposed for systems with rate-limited actuators.

\section{Conclusions}

In this paper, a real-life industrial control problem of compression systems was studied. The system is a multi-input multi-output process and, as is common industrial control processes, the actuators exhibit dynamic and static constraints. If these actuator constraints are not properly addressed, the closed-loop dynamics can be severely impaired due to integral windup in the controllers. The anti-windup method proposed in this paper not only ensures the avoidance of integral windup, but it can also be straightforward integrated in pre-existing control schemes without need of re-tuning. This becomes particularly useful when designing nonlinear controllers or adaptive algorithms, such as the varying PI strategy designed in this paper. The presented tuning of the PI controllers has considered actuator constraints. 

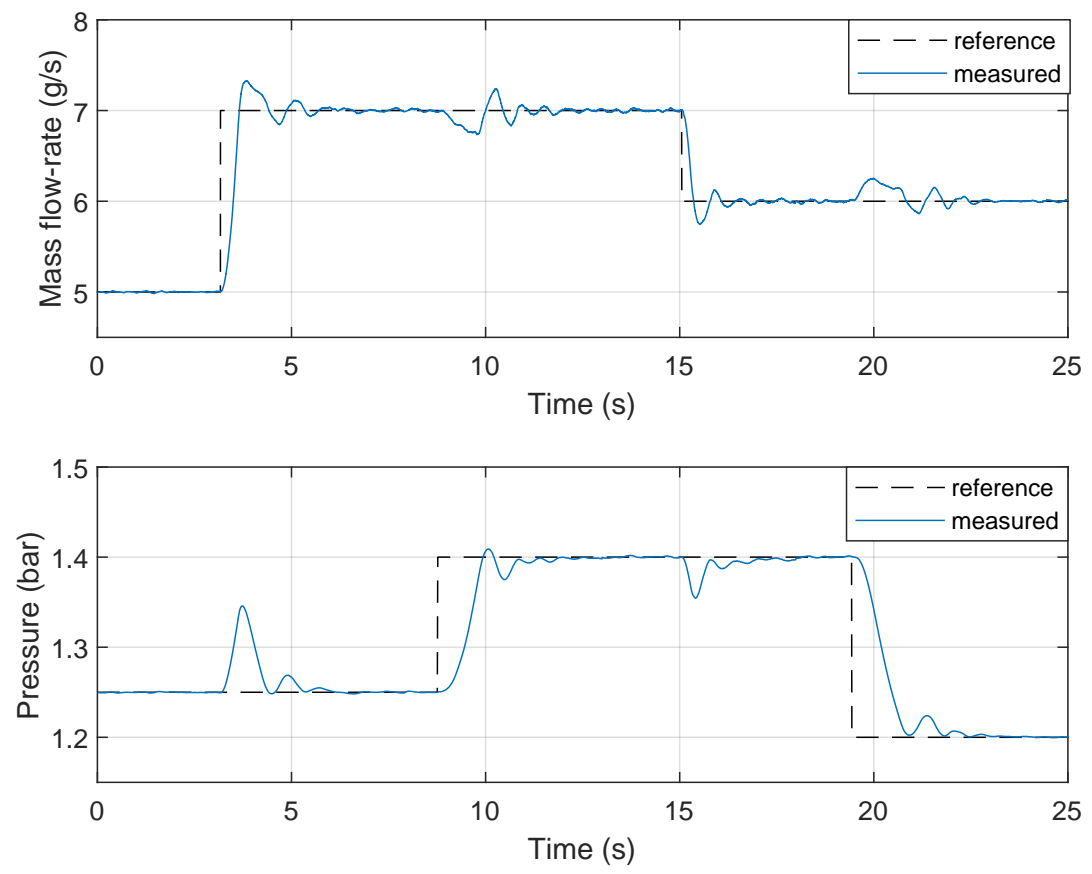

Figure 14: Experimental result of adaptive PI with AW

Both the simulation and the experimental results show that the integrated strategy of adaptive PI and anti-windup algorithms lead good control performance. However, it should be noted that since the actuators slew-rates are different in the case study of the automotive compressor, the speed of one loop becomes faster than the other. As a result, changes in the setpoint of the faster loop are reflected on the output of the slower loop. Nevertheless, the identification algorithm discussed in this paper can be used to analyze and mitigate the cross coupling effect between the two loops. Following the proposed methodology in this paper, the control scheme can be directly implemented in different nonlinear systems with actuator constraints associated with real-world applications.

\section{Acknowledgments}

The work presented in this paper and all the associated tests done were conducted at Brose Fahrzeugteile GmbH \& Co. This project has received funding from the Fuel Cells and Hydrogen 2 Joint Undertaking under grant agreement No 735969. This Joint Undertaking receives support from the 
European Unions Horizon 2020 research and innovation programme and Hydrogen Europe and N.ERGHY. The work is also an outcome of the Generalitat de Catalunya industrial doctorate program.

\section{References}

[1] T. Nguyen, F. Jabbari, Output feedback controllers for disturbance attenuation with actuator amplitude and rate saturation, Automatica 36 (9) (2000) 1339-1346.

[2] W. Sun, H. Gao, O. Kaynak, Vibration isolation for active suspensions with performance constraints and actuator saturation, IEEE/ASME Transactions on Mechatronics 20 (2) (2015) 675-683.

[3] V. Kapila, K. Grigoriadis, Actuator Saturation Control, CRC Press, 2002.

[4] V. Kapila, W. M. Haddad, Actuator amplitude saturation control for systems with exogenous disturbances, in: Proceedings of American Control Conference. ACC (IEEE Cat. No. 98CH36207), Vol. 3, 1998, pp. 1468-1472.

[5] J. Yuan, Y. Chen, S. Fei, Analysis of actuator rate limit effects on first-order plus time-delay systems under fractional-order proportionalintegral control, IFAC-PapersOnLine 51 (4) (2018) 37-42.

[6] J. Sofrony, M. C. Turner, I. Postlethwaite, Anti-windup synthesis for systems with rate-limits using riccati equations, International Journal of Control 83 (2) (2010) 233-245.

[7] J. M. Maciejowski, Predictive Control with Constraints, Pearson education, 2002.

[8] M. Morari, J. H. Lee, Model predictive control: Past, present and future, Computers \& Chemical Engineering 23 (4-5) (1999) 667-682.

[9] G. Goodwin, M. M. Seron, J. A. De Doná, Constrained Control and Estimation: an Optimisation Approach, Springer Science \& Business Media, 2006. 
[10] X. Yu-Geng, L. De-Wei, L. Shu, Model predictive control status and challenges, Acta Automatica Sinica 39 (3) (2013) 222-236.

[11] J. H. Lee, Model predictive control: Review of the three decades of development, International Journal of Control, Automation and Systems 9 (3) (2011) 415.

[12] M. L. Darby, M. Nikolaou, MPC: Current practice and challenges, Control Engineering Practice 20 (4) (2012) 328-342.

[13] M. G. Forbes, R. S. Patwardhan, H. Hamadah, R. B. Gopaluni, Model predictive control in industry: Challenges and opportunities, IFACPapersOnLine 48 (8) (2015) 531-538.

[14] A. Ferramosca, D. Limón, I. Alvarado, T. Alamo, E. F. Camacho, MPC for tracking with optimal closed-loop performance, in: 2008 47th IEEE Conference on Decision and Control, IEEE, 2008, pp. 4055-4060.

[15] F. Allgöwer, A. Zheng, Nonlinear Model Predictive Control, Vol. 26, Birkhäuser, 2012.

[16] K. Alexis, G. Nikolakopoulos, A. Tzes, Switching model predictive attitude control for a quadrotor helicopter subject to atmospheric disturbances, Control Engineering Practice 19 (10) (2011) 1195-1207.

[17] A. Di Giorgio, F. Liberati, S. Canale, Electric vehicles charging control in a smart grid: A model predictive control approach, Control Engineering Practice 22 (2014) 147-162.

[18] J. S. Lim, J.-S. Kim, Y. I. Lee, Robust tracking model predictive control for input-constrained uncertain linear time invariant systems, International Journal of Control 87 (1) (2014) 120-130.

[19] F. Garelli, R. J. Mantz, H. Battista, Advanced Control for Constrained Processes and Systems, Vol. 75, The Institution of Engineering and Technology, 2011.

[20] S. Tarbouriech, I. Queinnec, M. C. Turner, Anti-windup design with rate and magnitude actuator and sensor saturations, in: 2009 European Control Conference (ECC), IEEE, 2009, pp. 330-335. 
[21] A. Palmeira, J. Gomes da Silva Jr, S. Tarbouriech, I. Ghiggi, Sampleddata control under magnitude and rate saturating actuators, International Journal of Robust and Nonlinear Control 26 (15) (2016) 32323252 .

[22] S. Tarbouriech, T. Loquen, C. Prieur, Anti-windup strategy for reset control systems, International Journal of Robust and Nonlinear Control 21 (10) (2011) 1159-1177.

[23] J. Biannic, L. Burlion, S. Tarbouriech, G. Garcia, On dynamic inversion with rate limitations, in: American Control Conference (ACC), IEEE, 2012, pp. 191-196.

[24] I. Kolmanovsky, E. Garone, S. Di Cairano, Reference and command governors: A tutorial on their theory and automotive applications, in: American Control Conference, IEEE, 2014, pp. 226-241.

[25] U. Kalabic, I. Kolmanovsky, J. Buckland, E. Gilbert, Reference and extended command governors for control of turbocharged gasoline engines based on linear models, in: 2011 IEEE International Conference on Control Applications (CCA), IEEE, 2011, pp. 319-325.

[26] M. M. Nicotra, E. Garone, I. V. Kolmanovsky, Fast reference governor for linear systems, Journal of guidance, control, and dynamics 40 (2) (2016) 461-465.

[27] K. Kogiso, K. Hirata, A reference governor in a piecewise state affine function, in: 42nd IEEE International Conference on Decision and Control, Vol. 2, IEEE, 2003, pp. 1747-1752.

[28] K. Kogiso, K. Hirata, Reference governor for constrained systems with time-varying references, in: IEEE International Conference on Multisensor Fusion and Integration for Intelligent Systems, IEEE, 2006, pp. 359-364.

[29] A. Casavola, E. Mosca, D. Angeli, Robust command governors for constrained linear systems, IEEE Transactions on Automatic Control 45 (11) (2000) 2071-2077. 
[30] E. G. Gilbert, C.-J. Ong, Constrained linear systems with hard constraints and disturbances: An extended command governor with large domain of attraction, Automatica 47 (2) (2011) 334-340.

[31] F. Garelli, P. Camocardi, R. J. Mantz, Variable structure strategy to avoid amplitude and rate saturation in pitch control of a wind turbine, International Journal of Hydrogen Energy 35 (11) (2010) 5869-5875.

[32] K. Aström, T. Hägglund, PID Controllers: Theory, Design and Tuning, Instrument Society of America, 1995.

[33] I. Boiko, Non-parametric Tuning of PID Controllers, Springer, 2013.

[34] C. C. Yu, Autotuning of PID controllers: a relay feedback approach, Springer Science \& Business Media, 2006.

[35] Y. Eldigair, C. Kunusch, C. Ocampo-Martinez, P. Camocardi, Sliding mode anti-windup strategy for mass flow-rate regulation of compressors with rate-limited motors, in: 15th International Workshop on Variable Structure Systems (VSS), IEEE, 2018, pp. 378-383.

[36] P. C. Perera, Sensorless Control of Permanent-magnet Synchronous Motor Drives, Institute of Energy Technology, Aalborg University, 2002.

[37] D. Zhang, J. L. Di Liberti, M. Cave, Blade thickness effect on impeller slip factor, GT2010-22164, Proceedings of ASME Turbo Expo, Glasgow, UK.

[38] F. Hellström, Numerical computations of the unsteady flow in turbochargers, Ph.D. thesis, KTH (2010).

[39] O. Leufven, Compressor modeling for control of automotive two stage turbochargers, Ph.D. thesis, Linkopings Universitet (2010).

[40] T. S. Grong, Modeling of compressor characterisics and active surge control, Master's thesis, Institutt for Teknisk Kybernetikk (2009).

[41] J. T. Pukrushpan, A. G. Stefanopoulou, H. Peng, Control of Fuel Cell Power Systems: Principles, Modeling, Analysis and Feedback Design, Springer Science \& Business Media, 2004. 
[42] P. Moraal, I. Kolmanovsky, Turbocharger modeling for automotive control applications, Tech. rep., SAE Technical Paper (1999).

[43] S. Tarbouriech, M. Turner, Anti-windup design: An overview of some recent advances and open problems, IET Control Theory \& Applications 3 (1) (2009) 1-19.

\section{Appendix}


Table 2: Simulation values of $K_{c}\left(\times 10^{8}\right)$ for mass flow-rate loop

\begin{tabular}{|l|c|c|c|c|}
\hline & \multicolumn{4}{|c|}{ Pressure ratio } \\
\hline \multirow{4}{*}{$\begin{array}{l}\text { Mass } \\
\text { flow-rate } \\
\text { (g/s) }\end{array}$} & & $\mathbf{1 . 2}$ & $\mathbf{1 . 3}$ & $\mathbf{1 . 4}$ \\
\cline { 2 - 5 } & $\mathbf{5}$ & 1.92 & - & - \\
\cline { 2 - 5 } & $\mathbf{6}$ & 1.41 & 2.02 & - \\
\cline { 2 - 5 } & $\mathbf{7}$ & - & 1.58 & 2.28 \\
\cline { 2 - 5 } & $\mathbf{8}$ & - & 1.19 & 1.90 \\
\hline
\end{tabular}

Table 3: Simulation values of $K_{i}\left(\times 10^{8}\right)$ for mass flow-rate loop

\begin{tabular}{|l|c|c|c|c|}
\hline & \multicolumn{4}{|c|}{ Pressure ratio } \\
\hline \multirow{4}{*}{$\begin{array}{l}\text { Mass } \\
\text { flow-rate } \\
\text { (g/s) }\end{array}$} & & $\mathbf{1 . 2}$ & $\mathbf{1 . 3}$ & $\mathbf{1 . 4}$ \\
\cline { 2 - 5 } & $\mathbf{5}$ & 12.0 & - & - \\
\cline { 2 - 5 } & $\mathbf{6}$ & 7.85 & 14.4 & - \\
\cline { 2 - 5 } & $\mathbf{7}$ & - & 10.2 & 17.6 \\
\cline { 2 - 5 } & $\mathbf{8}$ & - & 6.91 & 13.4 \\
\hline
\end{tabular}

Table 4: Simulation values of $K_{c}\left(\times 10^{-5}\right)$ for pressure loop

\begin{tabular}{|l|c|c|c|c|}
\hline & \multicolumn{4}{|c|}{ Pressure ratio } \\
\hline \multirow{4}{*}{$\begin{array}{l}\text { Mass } \\
\text { flow-rate } \\
(\mathrm{g} / \mathrm{s})\end{array}$} & & $\mathbf{1 . 2}$ & $\mathbf{1 . 3}$ & $\mathbf{1 . 4}$ \\
\cline { 2 - 5 } & $\mathbf{5}$ & 5.13 & - & - \\
\cline { 2 - 5 } & $\mathbf{6}$ & 5.97 & 3.41 & - \\
\cline { 2 - 5 } & $\mathbf{7}$ & - & 3.93 & 2.54 \\
\cline { 2 - 5 } & $\mathbf{8}$ & - & 4.33 & 2.91 \\
\hline
\end{tabular}

Table 5: Simulation values of $K_{i}\left(\times 10^{-5}\right)$ for pressure loop

\begin{tabular}{|l|c|c|c|c|}
\hline & \multicolumn{4}{|c|}{ Pressure ratio } \\
\hline \multirow{4}{*}{$\begin{array}{l}\text { Mass } \\
\text { flow-rate } \\
(\mathrm{g} / \mathrm{s})\end{array}$} & $\mathbf{1 . 2}$ & $\mathbf{1 . 3}$ & $\mathbf{1 . 4}$ \\
\cline { 2 - 5 } & $\mathbf{5}$ & 3.95 & - & - \\
\cline { 2 - 5 } & $\mathbf{6}$ & 4.54 & 2.60 & - \\
\cline { 2 - 5 } & $\mathbf{7}$ & - & 3.02 & 1.89 \\
\cline { 2 - 5 } & $\mathbf{8}$ & - & 3.27 & 2.20 \\
\hline
\end{tabular}


Table 6: Experimental values of $K_{c}\left(\times 10^{7}\right)$ for mass flow-rate loop

\begin{tabular}{|l|c|c|c|c|}
\hline & \multicolumn{4}{|c|}{ Pressure ratio } \\
\hline \multirow{4}{*}{$\begin{array}{l}\text { Mass } \\
\text { flow-rate } \\
(\mathrm{g} / \mathrm{s})\end{array}$} & & $\mathbf{1 . 2}$ & $\mathbf{1 . 3}$ & $\mathbf{1 . 4}$ \\
\cline { 2 - 5 } & $\mathbf{5}$ & 3.85 & - & - \\
\cline { 2 - 5 } & $\mathbf{6}$ & 2.65 & 2.85 & - \\
\cline { 2 - 5 } & $\mathbf{7}$ & - & 2.58 & 2.04 \\
\cline { 2 - 5 } & $\mathbf{8}$ & - & 2.24 & 1.88 \\
\hline
\end{tabular}

Table 7: Experimental values of $K_{i}\left(\times 10^{8}\right)$ for mass flow-rate loop

\begin{tabular}{|l|c|c|c|c|}
\hline & \multicolumn{4}{|c|}{ Pressure ratio } \\
\hline \multirow{4}{*}{$\begin{array}{l}\text { Mass } \\
\text { flow-rate } \\
(\mathrm{g} / \mathrm{s})\end{array}$} & & $\mathbf{1 . 2}$ & $\mathbf{1 . 3}$ & $\mathbf{1 . 4}$ \\
\cline { 2 - 5 } & $\mathbf{5}$ & 3.98 & - & - \\
\cline { 2 - 5 } & $\mathbf{6}$ & 2.56 & 3.14 & - \\
\cline { 2 - 5 } & $\mathbf{7}$ & - & 2.55 & 1.78 \\
\cline { 2 - 5 } & $\mathbf{8}$ & - & 2.06 & 1.89 \\
\hline
\end{tabular}

Table 8: Experimental values of $K_{c}\left(\times 10^{-5}\right)$ for pressure loop

\begin{tabular}{|l|c|c|c|c|}
\hline & \multicolumn{4}{|c|}{ Pressure ratio } \\
\hline \multirow{4}{*}{$\begin{array}{l}\text { Mass } \\
\text { flow-rate } \\
(\mathrm{g} / \mathrm{s})\end{array}$} & $\mathbf{1 . 2}$ & $\mathbf{1 . 3}$ & $\mathbf{1 . 4}$ \\
\cline { 2 - 5 } & $\mathbf{5}$ & 3.36 & - & - \\
\cline { 2 - 5 } & $\mathbf{6}$ & 3.93 & 2.56 & - \\
\cline { 2 - 5 } & $\mathbf{7}$ & - & 3.15 & 2.28 \\
\cline { 2 - 5 } & $\mathbf{8}$ & - & 2.65 & 3.04 \\
\hline
\end{tabular}

Table 9: Experimental values of $K_{i}\left(\times 10^{-5}\right)$ for pressure loop

\begin{tabular}{|l|c|c|c|c|}
\hline & \multicolumn{4}{|c|}{ Pressure ratio } \\
\hline \multirow{4}{*}{$\begin{array}{l}\text { Mass } \\
\text { flow-rate } \\
(\mathrm{g} / \mathrm{s})\end{array}$} & & $\mathbf{1 . 2}$ & $\mathbf{1 . 3}$ & $\mathbf{1 . 4}$ \\
\cline { 2 - 5 } & $\mathbf{5}$ & 4.29 & - & - \\
\cline { 2 - 5 } & $\mathbf{6}$ & 6.97 & 2.87 & - \\
\cline { 2 - 5 } & $\mathbf{7}$ & - & 6.05 & 3.05 \\
\cline { 2 - 5 } & $\mathbf{8}$ & - & 4.99 & 5.99 \\
\hline
\end{tabular}

